\title{
Neospora caninum infection in birds: experimental infections in chicken and embryonated eggs
}

\author{
P. I. FURUTA, T. W. P. MINEO, A. O. T. CARRASCO, G. S. GODOY, A. A. PINTO \\ and R. Z. MACHADO* \\ Departamento de Patologia Veterinária, Faculdade de Ciências Agrárias e Veterinárias (FCAV), Universidade Estadual \\ Paulista Fúlio de Mesquita Filho (UNESP), Via de Acesso Prof. Paulo Donato Castellane s/n, Campus de Faboticabal, \\ Faboticabal, São Paulo State, Brazil
}

(Received 17 Fanuary 2007; revised 10 May and 9 Fune 2007; accepted 18 June 2007; first published online 9 August 2007)

\begin{abstract}
S U M MAR Y
Neospora caninum causes economical impact in cattle-raising farms since it is implicated as the major cause of bovine abortions. Although infection by the parasite has been widely described in mammals, the role of birds in its life-cycle is still obscure. Therefore, this work aimed to evaluate the infection by N. caninum in different chicken models. Experimental infections were conducted in 7-day-old chicks, laying hens and embryonated eggs, where samples were analysed for parasite burden, IgG antibodies and lesions promoted. Chickens demonstrated an asymptomatic infection, although with seroconversion and systemic replication of the parasite. In laying hens, no signs of vertical transmission were observed. However, embryonated eggs inoculated by the allantoic cavity route demonstrated susceptibility to infection, with mortality rates around $50 \%$ independent of the inoculum dose. Additionally, dogs became infected after ingestion of different amounts of inoculated eggs, producing either oocysts or specific IgG antibodies. The results herein presented demonstrate that chickens may be intermediate hosts of $N$. caninum and that embryonated eggs could be a useful model to study the parasite's biology.
\end{abstract}

Key words: Neospora caninum, chicken, embryonated eggs, laying hens, dogs, experimental infections.

\section{INTRODUCTION}

Neospora caninum, an obligatory intracellular protozoan, causes economical impact in cattle-raising farms due to reproductive disorders, and is implicated as the major identifiable cause of bovine abortions (Reichel and Ellis, 2006). Since its first description (Bjerkås et al. 1984), an extensive number of species have been investigated for their role in the parasite's life-cycle. Canids, represented by domestic dogs and coyotes, have been implicated as its definitive hosts, due to fecal oocyst shedding (McAllister et al. 1998; Gondim et al. 2004), and canine seroprevalences may reach up to $97 \%$ in some populations worldwide (Barber et al. 1997). Environment-contaminating oocysts are responsible for disseminating the protozoan, and the parasite is maintained inside herds through endogenous or exogenous transplacental infection (Trees and Williams, 2005), where serological prevalences of different herds may vary from low percentages to as high as 50\% (Wouda et al. 1999; Bartels et al. 2006; Mineo et al. 2006). Additionally, infection or prior

* Corresponding author: Immunoparasitology Laboratory, Department of Veterinary Pathology, FCAV/ UNESP, Jaboticabal, SP, Brazil 14.884-900. Tel: +55 163209 2662. E-mail: zacarias@fcav.unesp.br exposure has been detected in other domestic and wild animals, and questions are still raised about the zoonotic potential of the parasite (Dubey et al. 2007).

Although N. caninum has been widely described in mammals, the role of birds in the parasite's life-cycle is still obscure. It is known that birds are preyed upon by canids, and could be a good source of infection to the parasite's definitive hosts (McGuire et al. 1999; Gondim, 2006). Also, pigeons and zebra finches were shown to be potential intermediate hosts of the parasite, since experimental infections have established that birds may be susceptible to infection by the protozoan (McGuire et al. 1999). Before the definitive host description by McAllister et al. (1998), experimental infections were undertaken in carnivorous birds, however, no clinical signs of infection or oocysts were observed (Baker et al. 1995). Moreover, the presence of birds in cattleraising farms represents increased risk factors for abortion storms related to $N$. caninum (Bartels et al. 1999).

Since there is lack of information about avian species as hosts of $N$. caninum, this work aimed to evaluate the infection by the parasite in chickens, observing mortality, morbidity, vertical transmission and the use of these birds as an intermediate host model for the protozoan infection. 
MATERIALS AND METHODS

\section{Parasites}

The Neospora caninum NC-1 isolate (Dubey et al. 1988) was used for all experimental infections performed in this study. The parasites were cultured in African green monkey kidney cells (CV-1) and harvested by scraping off the cell monolayer 4-5 days after infection. To prepare inoculum doses, parasites were pelleted by centrifugation, concentration set with a Neubauer chamber, and diluted in RPMI medium (Sigma Co., USA) supplemented with antibiotic/antimycotic solution, containing penicillin (10 $000 \mathrm{U})$, streptomycin $(100 \mu \mathrm{g})$, and amphotericin $\mathrm{B}(25 \mu \mathrm{g})$ (Invitrogen, USA).

\section{Animals and experimental infections}

Chickens. Five groups, with ten 7-day-old male chicks each, were inoculated with 4 distinct concentrations of $N$. caninum tachyzoites $\left(10^{3}, 10^{4}, 10^{5}\right.$, $10^{6}$, and control), intraperitoneally. The animals were housed in separated boxes, with ration and water ad libitum. The experimental period was set at 60 days post-infection (p.i.), with programmed euthanasia of 3 animals/group at 15 days p.i. and at the end of the experiment. The remaining animals after the 15 day p.i. euthanasia were re-inoculated with $10^{7}$ tachyzoites/animal, as a trial for disease induction. Sera and samples of brain, heart, lungs, liver, spleen, eye and pectoral muscle were collected from all animals for further analysis, and mortality was assessed for each group.

Laying hens. Three laying hens of a commercial posture lineage, at 40 weeks of age, were experimentally inoculated with $10^{8} \mathrm{~N}$. caninum tachyzoites intraperitoneally, to evaluate vertical transmission by the cloacae-egg route in a 60-day experimental period. The hens were accommodated in individual cages with food and water supplied ad libitum. After inoculation, yolks from non-embryonated eggs produced by the hens, were centrifuged individually with $40 \mathrm{ml}$ of sterile PBS supplemented with $1 \%$ Tween 80 (GE Healthcare, USA) at $600 \boldsymbol{g}$, for $10 \mathrm{~min}$, at $4{ }^{\circ} \mathrm{C}$. The concentrated mass, which varied between 1 and $3 \mathrm{ml}$, was diluted 1:1 in sterile $\mathrm{PBS}$ and inoculated into Balb/c mice for bioassay ( $1 \mathrm{ml} /$ mice). Three mice were inoculated per egg/hen to observe if there was seroconversion due to the presence of live parasite forms or $N$. caninum antigens. After 30 days p.i., the mice were bled and serum submitted to IFAT protocol for the detection of specific anti- $N$. caninum $\operatorname{IgG}$ antibodies. Additionally, smears of the concentrated mass were analysed by light microscopy and assayed for the presence of parasite DNA by PCR. Sera were taken from the hens, by brachiocephalic punction, at 15 and 60 days p.i. to observe the presence of specific
IgG anti- $N$. caninum by IFAT. The animals were euthanized and samples of brain, heart, lungs, liver, spleen, eye and pectoral muscle were fixed in $10 \%$ buffered formal solution for histopathological and immunohistochemical examination.

Embryonated eggs. In Experiment 1 five groups containing 5 eggs/each were assayed with different $N$. caninum tachyzoite inoculums $\left(10^{3}, 10^{4}, 10^{5}, 10^{6}\right.$, and control). Eggs presented 10 days of incubation at 0 days p.i., and were maintained at an incubator with controlled temperature, humidity and rotation. An additional egg lot with 8 days of incubation was also inoculated with $10^{6}$ tachyzoites, to observe possible age differences in the infection outcome. Inoculation was performed in the chorioallantoic (CA) liquid, according to a previous description (Warren and Russ, 1948). The embryos were observed until hatching; however, prior mortality was recorded. Dead or euthanized embryos were necropsied and samples of brain, heart, lungs, liver, spleen, eye and pectoral muscle were collected for further histological and immunohistochemical analysis. Fragments of CA membranes and aliquots of CA liquid were stored at $-70{ }^{\circ} \mathrm{C}$ for subsequent PCR assays and gene expression assays. Experiment 2 consisted of the serial passage of $N$. caninum tachyzoites in CA liquid. Fifteen 10-day-old embryonated eggs were used in each passage trial. Firstly, the initial egg lot was inoculated with $10^{6} \mathrm{~N}$. caninum tachyzoites, and left for 7 days at an incubator. After that period, CA liquid was extracted from viable eggs, centrifuged at $1000 \mathrm{~g}$, at $4{ }^{\circ} \mathrm{C}$ for $10 \mathrm{~min}$, washed twice in sterile PBS and parasites were counted in a Neubauer chamber. Aliquots were stored at $-20{ }^{\circ} \mathrm{C}$ for subsequent PCR assays. A new 15 egg lot was subsequently inoculated and left for another 7-day incubation period until a new serial passage attempt. In Experiment 3 fifty embryonated eggs, with 10 days of incubation, were inoculated with $10^{6} \mathrm{~N}$. $\mathrm{ca}$ ninum tachyzoites/each. After 8 days p.i., embryos were euthanized and portions were separated for 3 different bioassay protocols. Assay 1 in which $1 \mathrm{dog}$ was fed once with 5 infected CA membranes, Assay 2 in which 2 dogs were fed with 2 whole infected eggs/ day (excluding egg shells), during 4 consecutive days and Assay 3 in which 4 dogs were fed each with 2 eggs/day (excluding egg shells), during 3 consecutive days, with feeding procedures being repeated at 15 and 30 days p.i. All tested dogs were followed up for oocyst shedding and $\operatorname{IgG}$ seroconversion, for at least 30 days. Serum samples were obtained from dogs by weekly brachiocephalic punction. Oocyst detection and estimates followed previously described protocols (Gondim et al. 2002). The dogs used in this experiment were cross bred (without a specific breed characteristic), with ages varying between 3 and 6 months, housed in individual boxes, with dry pelleted commercial ration (Royal Canin, 
France) and mineral water ad libitum. The animals were previously screened for the presence of antibodies to N. caninum, Toxoplasma gondii, Babesia canis, Ehrlichia canis, and Leishmania chagasi. Also, the puppies underwent de-worming (Praziquantel, Pyrantel embonate and Febantel), elimination of possible coccidial infections (Sulfadimethoxine and Metronidazole) and immunization protocols (canine distemper virus, parainfluenza, Leptospira interrogans, coronavirus, canine hepatitis, canine parvovirus) before being used in the bioassays described above. To eliminate the risk of cross-contamination between the above-described groups, each experiment was conducted separately, followed by thorough disinfection procedures with $2 \%$ sodium hypochlorite and $1 \%$ formaldehyde solutions, and at least 1 month vacant period.

All animal procedures were performed according to the Ethical Principles in Animal Research adopted by the Brazilian College of Animal Experimentation and to the 2000 Report of the AVMA Panel on Euthanasia (AVMA, 2001).

\section{Analysis of the obtained samples}

The indirect immunofluorescence antibody technique was used to determine the presence of $\mathrm{IgG}$ antibodies and $N$. caninum (IFAT-Nc) in the experimentally infected animals, following a previously described technique (Mineo et al. 2001), with some modifications for the chicken experimental model and the bioassayed mice. Briefly, the cut-off dilution for $\operatorname{dog}$ sera was set at $1: 25$, and an anti-dog IgG conjugated to fluorecein isothiocyanate (FITC - Sigma), diluted $1: 350$, was used to detect parasite-specific antibodies. The birds' sera were screened initially at $1: 20$ dilution, and anti-chicken IgG FITC conjugate (Sigma) was used, diluted 1:50. Mice sera had screening dilution set at $1: 25$, with the use of an antimouse IgG FITC conjugate (Sigma) as secondary antibody, with working dilution at $1: 100$. Buffers, washing protocols, and incubation periods were the same for all protocols. Only a bright fluorescence of the tachyzoite's entire surface was considered as a positive result. Positive samples were titrated until end-point dilution.

\section{Histopathological analysis and immunohistochemistry (IHC)}

Standard Haematoxylin and Eosin histostain (H\&E) and IHC assays were performed in paraffin-embedded tissues of experimentally infected chickens and eggs. For IHC, slides were assayed using 2 polyclonal anti- $N$. caninum sera, raised in experimentally infected calf and inbred Balb/c mice, as primary antibodies. The calf used was previously screened and found to be free of serum antibodies for Babesia bigemina, B. bovis, Anaplasma marginale,
Toxoplasma gondii, Brucella abortus and Leptospira interrogans. Furthermore, assays with both primary antibodies against $N$. caninum were carried out to ensure the assay's specificity, and exclude possible cross-reactions due to the presence of antibodies against other Apicomplexan parasites in the calf's serum, especially those which present difficult routine diagnosis and are frequently found in bovines, as Eimeria and Sarcocystis species. Immunohistochemical procedures were performed following previous description (van Maanen et al. 2004). Briefly, primary antibodies (anti-N. caninum) were incubated at $1: 1000$ dilution. The avidinbiotin complex immunoperoxidase step was performed (DakoCytomation, Denmark) and the slides stained with diaminobenzidine tetrahydrochloride (DAB - DakoCytomation). Counter-staining was performed with Harris's haematoxylin (10\%) and slides were mounted with cover-slips. The reaction was examined under a light microscope (Nikon).

\section{Nucleic acid isolation}

Total DNA was extracted from $5-8 \mathrm{ml}$ of yield CA liquid/egg and $25 \mathrm{mg}$ of nitrogen-macerated CA membranes. RNA from CA membranes was extracted from the same amount of tissue, also using grounding protocols with liquid nitrogen. Oocysts present in dog's feces were purified by sucrose gradient, washed twice in ultrapure water, and submitted to 6 freeze and thaw cycles. Afterwards, the samples were submitted to 1-min spinning cycles, using $0.5 \mathrm{~mm}$ glass beads to ensure exposure of oocyst contents. Extractions were performed using commercially available kits (DNeasy and RNeasy, Qiagen, USA), following the manufacturer's instructions.

\section{$R T-P C R$}

To observe parasite stage conversion inside egg membranes, the expression of messenger RNA (mRNA) for 2 genes was analysed: NcSAG1, a tachyzoite-specific marker (Howe et al. 1998); and bradyzoite specific NcSAG4 (Fernández-García et al. 2006). The reverse transcriptase reaction was designed following published protocol (FernándezGarcía et al. 2006). Culture-derived tachyzoites were used as controls in this experiment.

\section{PCR}

The resulting cDNAs were submitted to amplification by polymerase chain reaction, using specific primers (Table 1). The employed protocol was the same as that used to verify the presence of $N$. caninum DNA in CA liquid and membranes of infected eggs and oocysts shed by dogs by Nc5 gene amplification, realized as in the previous description (Yamane et al. 2000). 
Table 1. Oligonucleotides used for detection of parasite-specific DNA and tachyzoite- and bradyzoitespecific gene expression assays

\begin{tabular}{|c|c|c|c|c|}
\hline \multirow[b]{2}{*}{ Target gene } & \multirow[b]{2}{*}{ GeneBank ID } & \multicolumn{2}{|c|}{ Primers sequence $5^{\prime}$ to $3^{\prime}$} & \multirow{2}{*}{$\begin{array}{l}\text { Product } \\
\text { size (bp) }\end{array}$} \\
\hline & & Sense & Anti-sense & \\
\hline $\mathrm{Nc} S A G 1$ & AF132217 & gcaaggagataccgttggaa & gtttgcaccgtaagagcaca & 242 \\
\hline $\mathrm{Nc} S A G 4$ & AY763105 & caagttctcagggettcgtc & cagtgcaaaagcaagagctg & 291 \\
\hline Nc5 & X84238 & agtcaacctacgtcttct & gtgcgtccaatcctgtaac & 327 \\
\hline
\end{tabular}

RESULTS

\section{Chicken}

None of the 4 inoculated groups presented mortality of its animals during the experimental period or clinical variations after inoculation. At 15 days p.i., all euthanized animals presented 1:400 IgG antibody titres by IFAT, with parasites being detected in most sampled organs by IHC, showing disseminated acute infection by $N$. caninum. Reinfection of the animals did not produce any clinical or pathological alteration in the studied groups. At the end of the experiment, the sampled animals showed negative IFAT results in all groups, followed by the absence of parasites detectable by IHC in its tissues.

\section{Laying hens}

All three animals remained healthy during the 60-day observation period. Parasites were not found in the eggs by microscopy or PCR, and these results were further confirmed by negative mouse bioassays. IFAT results demonstrated a similar pattern to those observed in inoculated chickens, with $\mathrm{IgG}$ titres of $1: 400$ at 15 days p.i., and non-detectable specific antibodies at 60 days p.i.

\section{Mortality and cytopathic effects of $\mathrm{N}$. caninum infection in embryonated eggs}

The inoculated groups showed mortality between 18 and 21 days of incubation (7-10 days p.i.); however, there was no significant variation in embryo death according to inoculum dose (Fig. 1). Gross examination of the embryos and attached tissues showed dose-dependent effects of infection, although most eggs showed mainly haemorrhagic and thickened CA membranes with multifocal lesions (Fig. 2A). Some lesions associated to the embryo's heart and liver were also identified at gross examination. Eight-dayold egg embryos showed to be more susceptible to infection, since $100 \%$ of the assayed eggs in this group died before 18 days of incubation (10 days p.i.), with high parasite concentration and accentuated lesions in its tissues.
$\mathrm{H} \& \mathrm{E}$ histo-analysis of embryo tissues and attachments confirmed dose-dependent lesions, with the $10^{3}$ inoculated group presenting lesions mainly related to $\mathrm{CA}$ membranes, which showed congestion and focal inflammation with the presence of mononuclear and polymorphonuclear infiltrates. The $10^{4}$ group demonstrated lesions in the heart and liver of embryos, besides multifocal inflammation in CA membranes. When analysing the higher inoculum group, embryos presented necrosis in brain, heart, and liver, with parasitophagous vacuoles being identified in almost all tissues analysed. CA membranes of this last group were shown to be totally occupied by dividing parasites, with a high degree of inflammation, necrosis and haemorrhage (Fig. 2B). IHC assays confirmed the H\&E staining and gross examination, with parasites being detected in all sampled tissues (brain, spleen, heart, liver, skeletal muscles, intestines, and CA membranes), with a higher concentration of parasites being detected in the $10^{5}$ and $10^{6}$ inoculum dose groups (Fig. 2C). Amplification of the Nc5 gene also corroborates with the data presented herein, demonstrating the presence of $N$. caninum DNA in the CA liquid and membranes of all embryonated eggs used for this experiment. Expression of $\mathrm{Nc} S A G 1$ and $\mathrm{NcSAG} 4$ genes was assessed to observe parasite stage conversion inside embryonated eggs. Nc5 PCR-positive samples of CA membranes showed simultaneous expression of mRNA to both genes, with higher band intensity for $\mathrm{Nc} S A G 1$. However, a gradual increment in $\mathrm{NcS} A G 4$ expression was observed in embryos with longer incubation periods, associated with weakened band intensity for NcSAG1 (Fig. 3).

The surviving embryos that hatched after 21 days of incubation presented clinical signs compatible with neurological disorders, with chicks showing lack of coordination, pedalling movements or hind limb paralysis, and circular walking patterns. Only 2 of 11 chicks that hatched their shells (from lower dosage groups $-10^{3}$ and $10^{4}$ ) did not present clinical alterations due to infection. $\mathrm{H} \& \mathrm{E}$ and $\mathrm{IHC}$ analysis of these animals showed the presence of $N$. caninum in all analysed tissues, with a higher presence of parasites found in the brain, heart and liver. 

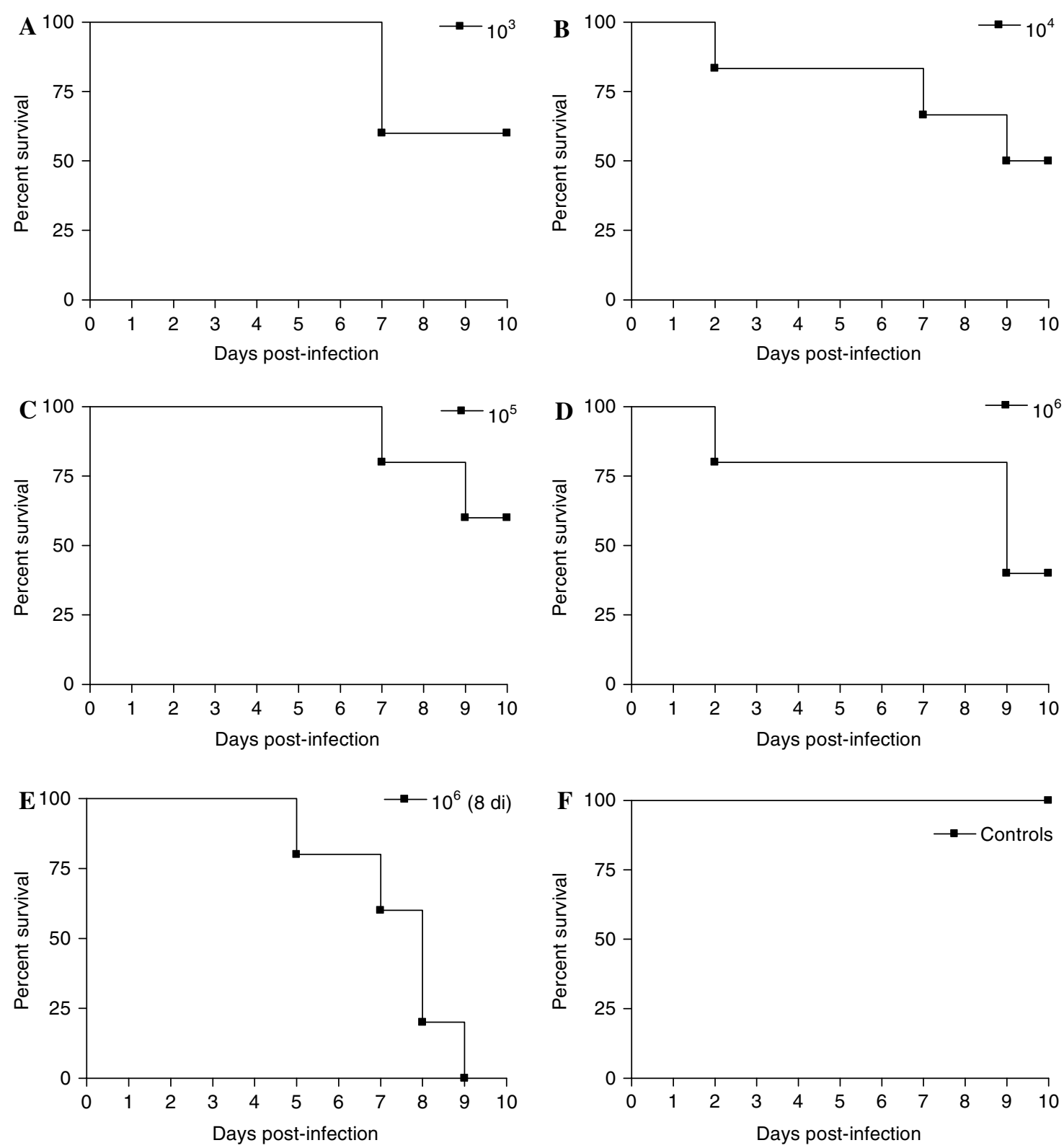

Fig. 1. Mortality rates in 10-day-old embryonated eggs experimentally infected with Neospora caninum tachyzoites, with different inoculum doses. (A) $10^{3}$ tachyzoites; (B) $10^{4}$ tachyzoites; (C) $10^{5}$ tachyzoites; (D) $10^{6}$ tachyzoites; (E) $10^{6}$ tachyzoites, inoculated into eggs, with an incubation period of 8 days; $(\mathrm{F})$ control group.

Serial passage of N. caninum tachyzoites in chorioallantoic liquid

After the first 7-day incubation period, 8 out of 15 eggs remained viable. CA liquid was collected from 6 of these eggs, since the other 2 eggs were discarded due to yolk rupture. Parasites were visualized in all collected samples, although at different intensities, being confirmed by PCR. A total of $7 \times 10^{6}$ parasites were retrieved from the 6 analysed eggs. The second passage attempt was not successful, since no parasites were visualized in CA liquid. PCR assays presented a low parasite yield in $3 / 10$ surviving eggs' CA liquid, but all presented positive PCR results in CA membranes, although with weak intensity. CA membranes were ground to free intracellular parasites, but pooled parasite yield was too low for a new passage attempt $\left(2 \times 10^{4}\right)$.

Ability of embryonated eggs to induce oocyst shedding in dogs

The dog fed once with highly parasitized CA membranes (Assay 1) shed N. caninum oocysts after 10 days p.i., for 5 consecutive days. During the elimination period, an estimated total shedding of 20000 oocysts was determined, although the animal remained serologically negative until 30 days p.i. In Assay 2, in which dogs were served whole eggs, lower oocyst shedding was observed in only $1 \mathrm{dog}$ at day 34 post-infection with 


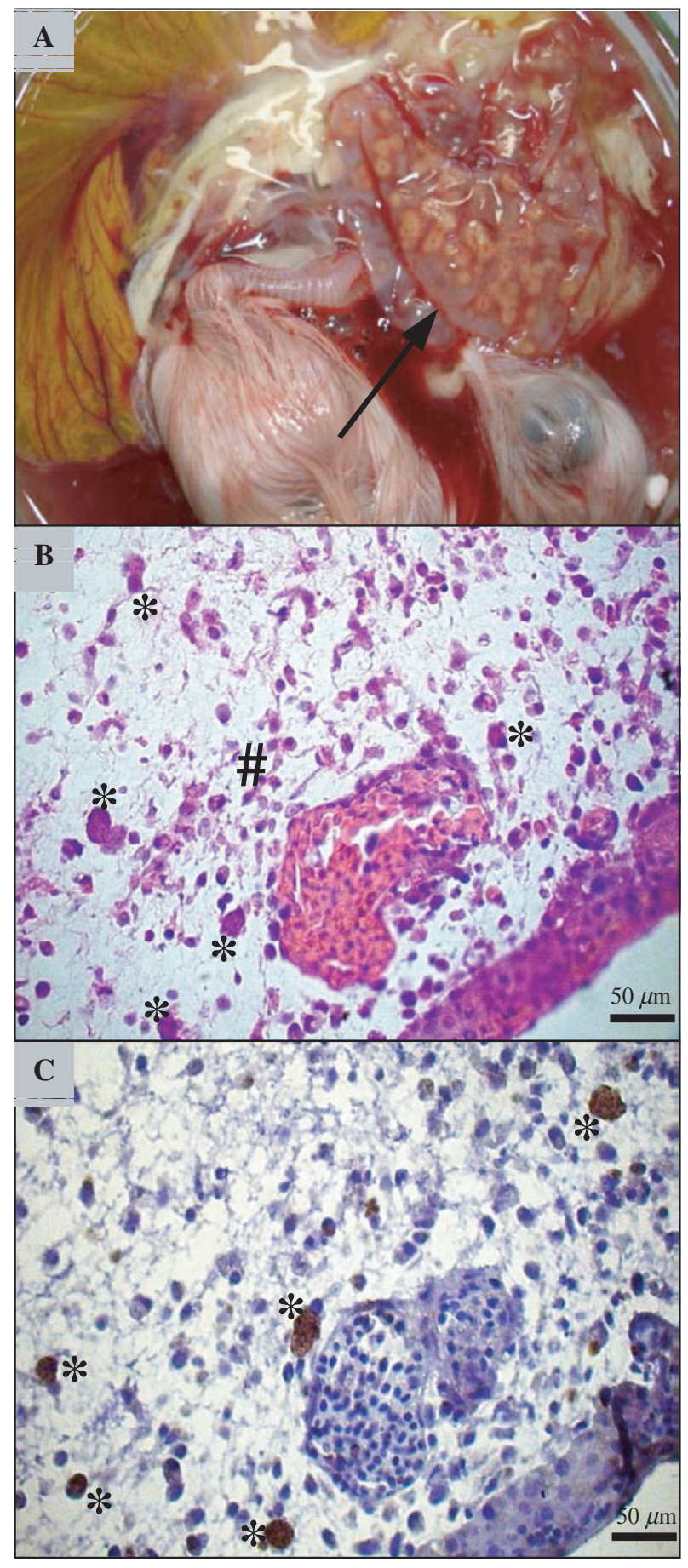

Fig. 2. (A) Eighteen-day-old chicken embryo experimentally infected with $10^{6}$ Neospora caninum NC-1 tachyzoites, presenting macroscopic lesions in its chorioallantoic membranes (arrow), viewed in (B) by $\mathrm{H} \& \mathrm{E}$ staining, demonstrating mixed inflammatory infiltrate $(\#)$ and parasite multiplication $(*)$, also revealed by IHC (C).

elimination estimated at 1000 oocysts, and no seroconversion was detected in that period. PCR analysis of eliminated oocysts revealed specific amplification of $N$. caninum's Nc5 gene (Fig. 4). There was no oocyst detection after 90 days p.i. in the 4 dogs used in Assay 3, where repeated

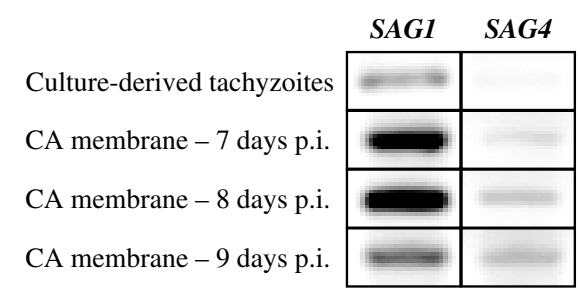

Fig. 3. Gene expression profile of Neospora caninum present in chorioallantoic membranes during infection of embryonated eggs, in different incubation periods.

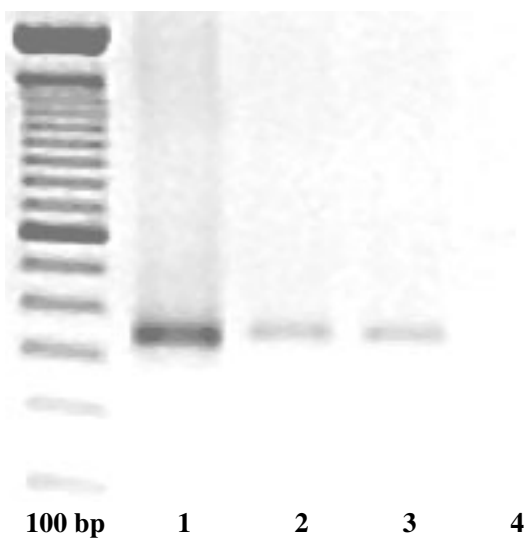

Fig. 4. PCR-positive amplification of Neospora caninum Nc5 gene (327 bp) in oocysts shed by the dog from Experiment 1 (lane 2) and Experiment 2 (lane 3). Reaction controls are represented by DNA extracted from $10^{7}$ culture-derived NC-1 tachyzoites (positive control in lane 1) and by amplified ultrapure water (negative control in lane 4).

infections occurred. However, $3 / 4$ dogs presented specific $\operatorname{IgG}$ antibodies to $N$. caninum after 45 days p.i., with titres ranging from $1: 25$ to $1: 400$ (Table 2).

\section{DISCUSSION}

Chickens seem to be resistant to infection by $N$. caninum, since no morbidity or mortality was observed in experimentally infected chickens and laying hens. Similar results were seen in broilers experimentally infected with $T$. gondii where no clinical modifications were observed, however the term 'resistance' was questioned by the authors, once they re-isolated parasites from diverse tissues after over 30 days p.i. (Kaneto et al. 1997). Specific IgG antibody synthesis was not parasite dosedependent, since antibody titres observed were identical in different inoculum groups. Similar titres were observed in chickens and laying hens, reaching IgG production peaks $(1: 400)$ at 15 days p.i., with negative antibody detection after 2 months of infection, even though high parasite doses were used to infect the animals. IHC assays showed that $N$. caninum was capable of multiplying and invading diverse host tissues. However, the animals 
Table 2. Seroconversion and oocyst detection pattern of dogs submitted to distinct protocols of Neospora caninum-infected embryonated eggs

\begin{tabular}{llll}
\hline \hline Ingestion & Dog & Oocyst detection & Seroconversion \\
\hline CA membranes & 1 & $+(20 \cdot 000$ oocysts, & - \\
Whole egg $(1 x)$ & 1 & $-11-16$ days p.i. $)$ & - \\
& 2 & $+(1000$ oocysts, & - \\
Whole egg $(3 x)$ & 1 & - & $+($ IgG titre $=1 / 100)$ \\
& 2 & - & - \\
& 3 & - & $+(\operatorname{IgG}$ titre $=1 / 25)$ \\
& 4 & - & $+(\operatorname{IgG}$ titre $=1 / 400)$ \\
\hline \hline
\end{tabular}

$(+)$ Positive samples.

(-) Negative samples.

demonstrated ability to control the infection, since parasite growth ceased before 60 days p.i. We suppose that $N$. caninum chronic infection in birds is represented by parasite migration to muscular tissues, where it remains in slow-growing tissue cysts. However, more detailed studies are necessary to describe the parasite's kinetics in this species. Also, there was no vertical passage between hens and eggs, since parasites were not detected inside non-embryonated eggs by various techniques. The cloacae-egg contact is an important transmission route of many avian pathogens such as Salmonella (Berchieri Junior et al. 2001). Laying hens experimentally infected with $T$. gondii oocysts did not present vertical transmission either, since the authors were unable to recover parasite forms from over 700 embryonated eggs (Biancifiori et al. 1986).

In order to evaluate if chicken embryos would be a suitable experimental model for Neospora infection, 3 main assays were performed in this study: a doseresponse curve, parasite serial passage trial, and capability of inducing dogs to behave as the parasite's definitive host. In the first assay, mortality was not shown to be related to inoculum dose, since all groups presented similar death patterns recorded after 7 days of infection. However, N. caninum induced greater lesions in embryos which received higher amounts of tachyzoites, as observed by macroscopical examination, H\&E histo-staining and IHC. Intense parasite multiplication in embryos that received $10^{5}$ and $10^{6}$ tachyzoites produced extensive inflammation throughout CA membranes, followed by widespread necrosis. The marked presence of parasitophagous vacuoles in embryo tissues was also noted, especially in the brain, heart and liver. Moreover, surviving embryos were born with neurological signs that resembled clinical neosporosis observed in mammalian species, such as dogs and calves. When analysing eggs inoculated at earlier incubation dates, N. caninum infection effects observed for 10 day old embryos were potentially increased, probably due to prolonged exposure of the parasite to embryos with immature immune responses, since immunological competence is reached only in the second half of the incubation period (Davidson, 2003). In that case, it is speculated that parasites divide themselves freely without any barrier imposed by the host, what would explain the higher mortality and lesion rates found in this group.

Embryonated eggs have been used over decades as a model for protozoan isolation (Buttitta, 1951), propagation (Warren and Russ, 1948; Roiron and Galistin, 1963; Wunderlin et al. 1997), and parasite biology studies (Mello and Deane, 1976; Que et al. 2004). The use of chicken embryos was a very feasible method to produce $T$. gondii antigens from the late 1940's until early 1970's, due to its low cost and sterile nature. To date, this experimental model has also the advantage of avoiding animal euthanasia (Wunderlin et al. 1997). Moreover, embryonated eggs may serve as a valuable tool to study different parasite stages, as seen with Trypanosoma cruzi, which displayed vertebrate and invertebrate specific stages inside egg's yolk and CA membranes (Mello and Deane, 1976).

Serial passage attempts of N. caninum in embryonated eggs failed after the first successful attempt, which produced about the same amount of the inoculum dose only in the CA liquid, excluding intracellular dividing parasites present in CA membranes and fetal tissues. However, the second passage gave out extremely low parasite yields, which made a further serial passage attempt impossible, due to the lack of tachyzoites. Parasites that undergo serial passages are generally used for live vaccine development, since virulence factors are attenuated, as recently shown for high passage $N$. caninum tachyzoites in cell culture (Bartley et al. 2006). Another interesting aspect observed in this experiment was that parasite forms found inside egg's CA membranes expressed simultaneously mRNA to tachyzoite and bradyzoite specific markers. That same gene expression profile was observed for culture-derived tachyzoites, which would lead 
to the idea that the infections in embryonated eggs would produce similar quality parasite yields as conventional in vitro protocols. However, an increase in bradyzoite-specific gene expression in eggs with longer incubation periods was also observed, which could mean that the appearance of tissue cysts is dependent on the infection time-span. A similar gene expression profile was observed previously (Fernández-García et al. 2006), where the authors justified their findings with a previous report from their research group, in which they affirmed that stage conversion is a progressive event (Risco-Castillo et al. 2004). A more refined research study would be suitable to evaluate $N$. caninum gene expression during invasion/evasion events, and embryonated eggs could be employed for that purpose.

In relation to the use of chicken embryos and its tissues to induce oocyst shedding in dogs, this work has shown that the most successful protocol applied was CA membrane intake by dogs. The amount of oocysts produced by the dog was not high; however, an improved standardized infection procedure may provide better results. The low operational costs and straightforward infected tissue production are positive points for this model, in contrast to major setbacks presented in experimental infections with other animal species, especially bovines. Additionally, infected CA membranes should mimic, at least in part, placental tissue often indicated as a source of parasites to canids. The dogs that underwent inoculation protocols using whole eggs also became infected, as proven by the presence of parasites in their feces or by $\operatorname{IgG}$ seroconversion. In that sense, dogs seem to have a delayed or deficient seroconversion to $N$. caninum, since specific $\mathrm{IgG}$ production is detected the earliest after 20-30 days in experimental infections, and specific antibodies against the parasite may be identified in serum samples only after several months, when detected at all (McAllister et al. 1998; Schares et al. 2001; McGarry et al. 2003; Gondim et al. 2005). Moreover, we may infer that dogs fed with repetitive parasite doses might have generated some kind of protective mucosal immunity towards oocyst shedding or, at least, delayed the event. Our research group is currently working on those subjects, aiming at the parasite intestinal cycle and canine immunity against $N$. caninum.

Finally, chickens may be a good intermediate host model for $N$. caninum, since the animals are partially resistant to infection: there is no clinical alteration in animals infected with high parasite doses, but parasites are able to infect a large variety of the animal's tissues. The results herein presented also suggest that chicken embryos and attached tissues may be of good use to study $N$. caninum's biology in living organisms, and also could be administered as inoculum to dogs for oocyst production, especially because of the low costs of model maintenance.
All embryonated eggs and 1-day chicks where furnished by Hy-line. This project was financially supported by the Fundação de Amparo a Pesquisa do Estado de São Paulo (FAPESP).

\section{REFERENCES}

American Veterinary Medical Association (2003). Report of the AVMA panel on Euthanasia. Fournal of the American Veterinary Medical Association 218, 669-696.

Baker, D. G., Morishita, T. Y., Brooks, D. L., Shen, S. K., Lindsay, D. S. and Dubey, J. P. (1995). Experimental oral inoculations in birds to evaluate potential definitive hosts of Neospora caninum. Fournal of Parasitology 81, 783-785.

Barber, J. S., Gasser, R. B., Ellis, J. T., Reichel, M. P., McMillan, D. and Trees, A. J. (1997). Prevalence of antibodies to Neospora caninum in different canid populations. Fournal of Parasitology 83, 1056-1058.

Bartels, C. J. M., Wouda, W. and Schukken, Y. H. (1999). Risk factors for Neospora caninum-associated abortion storms in dairy herds in The Netherlands (1995-1997). Theriogenology 52, 247-257.

Bartels, C. J. M., Arnaiz-Seco, J. I., RuizSanta-Quitera, A., Björkman, C., Frössling, J., von Blumröder, D., Conraths, F. J., Schares, G., van Maanen, C., Wouda, W., Ortega-Mora, L. M. (2006). Supranational comparison of Neospora caninum seroprevalences in cattle in Germany, The Netherlands, Spain and Sweden. Veterinary Parasitology 137, 17-27.

Bartley, P. M., Wright, S., Sales, J., Chianini, F., Buxton, D. and Innes, E. A. (2006). Long-term passage of tachyzoites in tissue culture can attenuate virulence of Neospora caninum in vivo. Parasitology 133, 421-432.

Berchieri Junior, A., Murphy, A., Marston, K. and Barrow, P. A. (2001). Observations on the persistence and vertical transmission of Salmonella enterica serovars Gallinarum and Pullorum in chickens: effect of bacterial and host genetic background. Avian Pathology 30, 221-231.

Biancifiori, F., Rondini, C., Grelloni, V. and Frescura, T. (1986). Avian toxoplasmosis: experimental infection of chicken and pigeon. Comparative Immunology, Microbiology and Infectious Diseases 9, 337-346.

Bjerkås, I., Mohn, S. F. and Presthus, J. (1984). Unidentified cyst-forming sporozoon causing encephalomyelitis and myositis in dogs. Zeitschrift für Parasitenkunde 70, 271-274.

Buttitta, P. L. (1951). Biology of Toxoplasma hominis in the guinea pig, mouse and embryonated egg. Bollettino della Società Italiana di Biologia Sperimentale 27, 83-84.

Davidson, T. F. (2003). The immunologists' debt to the chicken. British Poultry Science 44, 6-21.

Dubey, J. P., Carpenter, J. L., Speer, C. A., Topper, M. J. and Uggla, A. (1988). Newly recognized fatal protozoan disease of dogs. Fournal of the American Veterinary Medical Association 192, 1269-1285.

Dubey, J. P., Schares, G. and Ortega-Mora, L. M. (2007). Epidemiology and control of neosporosis and Neospora caninum. Clinical and Microbiological Reviews 20, 323-367.

Fernández-García, A., Risco-Castillo, V., Zaballos, A., Álvarez-García, G. and Ortega-Mora, L. M. (2006). Identification and molecular cloning of the 
Neospora caninum SAG4 gene specifically expressed at bradyzoite stage. Molecular and Biochemical Parasitology 146, 89-97.

Gondim, L. F. (2006). Neospora caninum in wildlife. Trends in Parasitology 22, 247-252.

Gondim, L. F., Gao, L. and McAllister, M. M. (2002). Improved production of Neospora caninum oocysts, cyclical oral transmission between dogs and cattle, and in vitro isolation from oocysts. Fournal of Parasitology 88, 1159-1163.

Gondim, L. F., McAllister, M. M. and Gao, L. (2005). Effects of host maturity and prior exposure history on the production of Neospora caninum oocysts by dogs. Veterinary Parasitology 134, 33-39.

Gondim, L. F., McAllister, M. M., Pitt, W. C. and Zemlicka, D. E. (2004). Coyotes (Canis latrans) are definitive hosts of Neospora caninum. International Fournal for Parasitology 34, 159-161.

Howe, D. K., Crawford, A. C., Lindsay, D. S. and Sibley, L. D. (1998). The p29 and p35 immunodominant antigens of Neospora caninum tachyzoites are homologous to the family of surface antigens of Toxoplasma gondii. Infection and Immunity 66, 5322-5328.

Kaneto, C. N., Costa, A. J., Paulillo, A. C., Moraes, F. R., Murakami, T. O. and Meireles, M. V. (1997). Experimental toxoplasmosis in broiler chicks. Veterinary Parasitology 69, 203-210.

McAllister, M. M., Dubey, J. P., Lindsay, D. S., Jolley, W. R., Willis, R. A. and McGuire, A. M. (1998). Dogs are definitive hosts of Neospora caninum. International Fournal for Parasitology 28, 1473-1478.

McGarry, J. W., Stockton, C. M., Williams, D. J. and Trees, A. J. (2003). Protracted shedding of oocysts of Neospora caninum by a naturally infected foxhound. Fournal of Parasitology 89, 628-630.

McGuire, A. M., McAllister, M. M., Wills, R. A. and Tranas, J. D. (1999). Experimental inoculation of domestic pigeons (Columbia livia) and zebra finches (Poephila guttata) with Neospora caninum tachyzoites. International Fournal for Parasitology 29, 1525-1529.

Mello, M. N. and Deane, M. P. (1976). Patterns of development of Trypanosoma cruzi in the embryonated chicken egg. Annals of Tropical Medicine and Parasitology 70, 380-388.

Mineo, T. W. P., Alenius, S., Näslund, K., Montassier, H. J. and Björkman, C. (2006). Distribution of antibodies against Neospora caninum, BVDV, and BHV1 among cows in Brazilian dairy herds with reproductive disorders. Brazilian Fournal of Veterinary Parasitology 15, 188-192.

Mineo, T. W. P., Silva, D. A. O., Costa, G. H. N., von Ancken, A. C., Kasper, L. H., Souza, M. A., Cabral, D. D., Costa, A. J. and Mineo, J. R. (2001). Detection of IgG antibodies to Neospora caninum and Toxoplasma gondii in dogs examined in a veterinary hospital from Brazil. Veterinary Parasitology 98, 239-245.

Que, X., Wunderlich, A., Joiner, K. A. and Reed, S. L. (2004). Toxopain-1 is critical for infection in a novel chicken embryo model of congenital toxoplasmosis. Infection and Immunity 72, 2915-2921.

Reichel, M. P. and Ellis, J. T. (2006). If control of Neospora caninum infection is technically feasible does it make economic sense? Veterinary Parasitology 142, 23-34.

Risco-Castillo, V., Fernández-García, A. and Ortega-Mora, L. M. (2004). Comparative analysis of stress agents in a simplified in vitro system of Neospora caninum bradyzoite production. Fournal of Parasitology 90, 466-470.

Roiron, V. and Galistin, P. (1963). Culture of Trichomonas vaginalis and Trichomonas foetus in the embryonated egg. Revue de Pathologie Générale et de Physiologie Clinique 63, 267-273.

Schares, G., Heydorn, A. O., Cuppers, A., Conraths, F. J. and Mehlhorn, H. (2001). Cyclic transmission of Neospora caninum: serological findings in dogs shedding oocysts. Parasitology Research 87, 873-877.

Trees, A. J. and Williams, D. J. L. (2005). Endogenous and exogenous transplacental infection in Neospora caninum and Toxoplasma gondii. Trends in Parasitology 21, 558-561.

van Maanen, C., Wouda, W., Schares, G., von Blumröder, D., Conraths, F. J., Norton, R., Williams, D. J., Esteban-Redondo, I., Innes, E. A., Mattsson, J. G., Björkman, C., Fernández-García, A., Ortega-Mora, L. M., Müller, N., Sager, H. and Hemphill, A. (2004). An interlaboratory comparison of immunohistochemistry and PCR methods for detection of Neospora caninum in bovine foetal tissues. Veterinary Parasitology 126, 351-364.

Warren, J. and Russ, S. B. (1948). Cultivation of toxoplasma in embryonated eggs - an antigen derived from chorioallantoic membrane. Proceedings of the Society for Experimental Biology and Medicine 67, 85-89.

Wouda, W., Bartels, C. J., Moen, A. R. (1999). Characteristics of Neospora caninum-associated abortion storms in diary herds in The Netherlands (1995 to 1997). Theriogenology 52, 233-245.

Wunderlin, E., Wild, P. and Eckert, J. (1997). Comparative reproduction of Cryptosporidium baileyi in embryonated eggs and in chickens. Parasitology Reasearch 83, 712-715.

Yamane, I., Kitani, H., Kokuho, T., Shibahara, T., Haritani, M., Hamaoka, T., Shimizu, S., Koiwai, M., Shimura, K. and Yokomizo, Y. (2000). The inhibitory effect of interferon gamma and tumour necrosis factor alpha on intracellular multiplication of Neospora caninum in primary bovine brain cells. Fournal of Veterinary Medical Sciences, Series B 62, 347-351. 